\title{
Desempenho reprodutivo de ovelhas submetidas a diferentes formas de suplementação alimentar antes e durante a estação de monta ${ }^{1}$
}

\section{Rinaldo Masato Mori ${ }^{2}$, Edson Luis de Azambuja Ribeiro ${ }^{3,4^{*}}$, Ivone Yurika Mizubuti ${ }^{3,4}$, Marco Antonio da Rocha ${ }^{3}$, Leandro das Dores Ferreira da Silva ${ }^{3}$}

\author{
${ }^{1}$ Parte da dissertação de Mestrado do primeiro autor apresentada à UEL. Projeto financiado pelo CNPq. \\ 2 Doutorando em Ciência Animal, UEL. \\ ${ }^{3}$ Departamento de Zootecnia da UEL, Londrina, PR. \\ ${ }^{4}$ Bolsista CNPq. \\ * Autor para correspondência.
}

\begin{abstract}
RESUMO - Objetivou-se com este estudo analisar o desempenho reprodutivo de ovelhas submetidas a diferentes formas de suplementação alimentar antes e durante o período de acasalamento. Utilizaram-se 112 ovelhas (63 no primeiro ano e 49 no segundo) dos grupos raciais Hampshire Down, Ile de France, Suffolk e Corriedale. Os animais foram separados em três grupos: T1 (sem suplementação), T2 (suplementação com $600 \mathrm{~g} / \mathrm{dia}$ de milho triturado) e T3 (suplementação com $600 \mathrm{~g} / \mathrm{dia}$ de concentrado constituído de $75 \%$ de milho triturado e $25 \%$ de farelo de soja). As ovelhas sob suplementação (T2 e T3) apresentaram maiores pesos e ganhos de peso até o final da estação de monta. Os escores da condição corporal também foram maiores ao final da estação de acasalamento nas ovelhas sob suplementação, implicando maior deposição de tecidos (músculo e gordura) no corpo do animal. O flushing não resultou em maior taxa de parição nem em aumento de partos gemelares, porém, ocasionou maior índice de natalidade nas ovelhas que receberam suplementação somente com milho. Os fatores idade e grupo racial das ovelhas afetaram significativamente o índice de natalidade. A suplementação energética com milho pode melhorar o número de cordeiros nascidos por ovelhas acasaladas.
\end{abstract}

Palavras-chave: escore da condição corporal, flushing, ovinos, reprodução

\section{Reproductive performance of ewes submitted to different feeding managements before and during the breeding period}

\begin{abstract}
The objective of this study was to investigate the reproductive performance of ewes submitted to different feeding management before and during the breeding period. A total of 112 ewes, 63 in year one and 49 in year two of the study, from the genetic groups Hampshire Down, Ile de France, Suffolk and Corriedale, were used. The ewes were separated in three groups: T1 (without supplementation), T2 (supplemented with $600 \mathrm{~g} /$ day of ground corn) and T3 (supplemented with 600 $\mathrm{g}$ /day of a concentrate containing $75 \%$ of ground corn and $25 \%$ of soybean meal). Supplemented ewes (T2 and T3) had higher body weight and more weight gain until the end of the breeding period than those not supplemented. Body condition scores were also greater at the end of the breeding period for supplemented ewes, indicating a greater fat and muscle deposition. Supplementation did not improve fertility rate and twins birth rate, but increased birth rate in ewes supplemented with corn. Age and genetic group both affected birth rate. It was concluded that feeding corn to ewes improved the number of lambs per ewe.
\end{abstract}

Key Words: body condition score, flushing, reproduction, sheep

\section{Introdução}

Atualmente, a atenção dos ovinocultores tem se voltado para a produção de carne, o que exige alta produtividade do rebanho, principalmente no que se refere à reprodução, visando aumentar a taxa de parição (número de ovelhas paridas por ovelhas acasaladas), a prolificidade (cordeiros nascidos por ovelha), o ganho de peso e a taxa de mortalidade (Selaive-Villarroel, 1986). Segundo Scaramuzzi \& Radford (1983), para melhorar a taxa de parição e a prolificidade, deve-se aumentar a taxa de ovulação, influenciada por vários fatores (Jainudeen \& Hafez, 1993), sobretudo a nutrição (Robinson et al., 2002).

A suplementação alimentar antes e durante o período de acasalamento (flushing alimentar) possibilita aumentos significativos nos parâmetros reprodutivos das ovelhas, em virtude do aumento da taxa de ovulação (Molle et al., 1997; Nottle et al., 1997, Branca et al., 2000) e da incidência de partos gemelares (Barioglio \& Rubiales de Barioglio, 1994; Mukasa-Mugerwa \& Lahlou-Kassi, 1995; Nottle et al., 
1997). Abecia et al. (1999) registraram taxa de prenhez de $100 \%$ em ovelhas submetidas ao flushing 15 dias após o início da estação de monta, enquanto naquelas sem suplementação essa taxa foi de $40 \%$. Adicionalmente, tem-se relatado que oflushing determina maior número de óvulos fertilizados, em razão do aumento da taxa de ovulação e da menor mortalidade embrionária (NRC, 1985).

O primeiro mês após a fertilização é crítico para a sobrevivência embrionária. Portanto, é importante continuar o flushing por 30 dias após a cobertura, tempo necessário para a implantação do embrião no útero (NRC, 1985).

Molle et al. (1995) sugeriram que a relação energia X proteína da dieta pré-acasalamento pode ser crítica para obtenção de uma resposta reprodutiva. De acordo com os autores, a suplementação energética por meio de grãos pode aumentar a taxa de ovulação, desde que a ingestão de proteína seja acima do limite mínimo requerido.

Este estudo foi realizado objetivando-se analisar o desempenho reprodutivo de ovelhas recebendo, antes e durante o período de acasalamento, milho triturado ou farelo de soja + milho triturado como suplementação alimentar.

\section{Material e Métodos}

O experimento foi conduzido na Fazenda Escola da Universidade Estadual de Londrina, situada em Londrina, Paraná $\left(23^{\circ} 20^{\prime} 10^{\prime \prime}\right.$ de latitude sul e $51^{\circ} 09^{\prime} 15^{\prime \prime}$ de longitude oeste), com $610 \mathrm{~m}$ de altitude, temperatura média anual de $20,6^{\circ} \mathrm{C}$ e precipitação média anual de $1.439,8 \mathrm{~mm}$, máxima em janeiro (201,4 mm)emínima emjunho(56,5 mm) (Maack, 1981).

Foram utilizadas 112 ovelhas (65 Hampshire Down, 22 Ile de France, 17 Suffolk e 8 Corriedale), sendo as Corriedale de raça definida e as demais, no mínimo, meio-sangue. Os animais foram divididos em três tratamentos (T1 - sem suplementação, T2 - suplementação com $600 \mathrm{~g}$ de milho triturado e T3 - suplementação com $600 \mathrm{~g}$ de uma mistura concentrada constituída de $75 \%$ de milho triturado e $25 \%$ de farelo de soja), segundo o grupo racial e a idade, durante dois anos de avaliação - 63 ovelhas em 2001 e 49 ovelhas em 2002 .

As estações de monta ocorreram no período de 15 de março a 30 de abril, totalizando 45 dias nos dois anos. Foi utilizada a monta natural, de modo que as ovelhas dos grupos raciais Hampshire Down, Ile de France e Suffolk foram acasaladas com cordeiros de suas respectivas raças e as Corriedale, com cordeiro Texel. Durante a estação de monta, as reprodutoras foram mantidas à noite com os cordeiros em um curral de piso concretado.

A suplementação alimentar teve início em 01 de março nos dois anos experimentais, totalizando 60 dias de suplementação em cada ano de avaliação. Nos três primeiros dias, foram fornecidos $400 \mathrm{~g}$ diários de suplemento, fracionados em duas vezes. A partir do quarto dia, o total fornecido foi de $600 \mathrm{~g} / \mathrm{dia}$, fracionados em duas vezes $(8 \mathrm{~h}$ e 17h30) até o início da estação de monta, quando o suplemento alimentar passou a ser fornecido somente pela manhã $(8 \mathrm{~h})$. Durante o dia, os animais foram mantidos em pastagem de coastcross (Cynodon dactylon (L.) Pers), em sistema rotacionado, com água e sal mineral à vontade. Da segunda quinzena de maio até o mês de novembro, as ovelhas passaram a receber silagem de milho (ano de 2001) ou sorgo (ano de 2002), fornecida ad libitum em comedouros no curral de manejo todos os dias à tarde (17h30). Todos os animais foram everminados ao início da suplementação alimentar e, posteriormente, de acordo com a contagem de ovos por grama de fezes.

Foram realizadas análises químicas para mensurar a composição em MS, PB, EE, FB, MM, FDN e FDA, segundo métodos de Weende e Van Soest, descritos por Silva \& Queiroz (2002) - das pastagens, dos suplementos alimentares e das silagens de milho e sorgo. Observou-se (Tabela 1) que os resultados analíticos da pastagem, do milho grão e do farelo de soja correspondem à média dos dois anos.

Foram mensurados o peso e o escore da condição corporal das ovelhas ao início da suplementação alimentar e ao início e final da estação de monta, os pesos das ovelhas ao parto (até 24 horas após o parto) e o número de cordeiros nascidos. O escore da condição corporal foi determinado segundo metodologia descrita por Caldeira \& Vaz Portugal (1998), atribuindo valores de 1 a 5 , em que 1 corresponde a animais muito magros e 5 , a animais obesos. Foram analisadas as taxas de parição (ovelhas paridas por ovelhas acasaladas) e de natalidade (cordeiros nascidos por ovelhas acasaladas) e a incidência de partos gemelares.

Tabela 1 - Composição química (\% na MS) dos alimentos utilizados nos dois anos experimentais

Table 1 - Average chemical composition (\% dry matter) of feeds used in the two experimental years

\begin{tabular}{lcccrr}
\hline Alimento (Feed) & MS (DM) & PB (CP) & EE & FDN (NDF) & FDA (ADF) \\
\hline Pastagem de coastcross (Coastcross pasture) & 42,73 & 10,46 & 1,45 & 79,36 & 37,36 \\
Silagem de milho (Corn silage) & 31,45 & 6,59 & 2,21 & 63,25 & 31,57 \\
Silagem de sorgo (Sorghum silage) & 30,21 & 7,52 & 2,09 & 68,47 & 4,91 \\
Milho triturado (Ground corn) & 90,07 & 9,06 & 4,52 & - & 33,52 \\
Farelo de soja (Soybean meal) & 90,27 & 46,21 & 0,88 & - & - \\
\hline
\end{tabular}


Os dados foram submetidos à análise de variância utilizando-se o programa estatístico SAS (SAS, 1994). Na análise de peso, ganho de peso e escore da condição corporal, foram considerados os efeitos da suplementação (antes e durante a estação de monta - tratamentos), do ano (2001 e 2002), da idade da ovelha (4, 6 e 8 dentes) e do grupo racial (Corriedale, Hampshire Down, Ile de France e Suffolk). Como não apresentaram diferenças significativas, as interações duplas entre os efeitos foram desconsideradas do modelo estatístico final. As diferenças entre médias foram testadas pelo teste $t$ a 5\% de significância e as taxas de parição e a incidência de partos gemelares nos diferentes tratamentos, anos, idades e grupos raciais foram analisadas pelo teste do qui-quadrado.

\section{Resultados e Discussão}

Na Tabela 2 são apresentadas as médias dos pesos das ovelhas em diferentes períodos de acordo com os diferentes parâmetros avaliados.
As ovelhas que receberam suplementação alimentar (T2 e T3) apresentaram peso corporal superior ao observado no tratamento sem suplementação (T1) somente ao final da estação de monta $(\mathrm{P}<0,01)$. O peso dos animais do tratamento sem suplementação manteve-se até o parto, enquanto o dos grupos com suplementação apresentou pequena redução do final da estação de acasalamento até o parto.

Ovelhas de oito dentes apresentaram pesos superiores aos daquelas de quatro e seis dentes $(\mathrm{P}<0,01)$ durante todo o período de suplementação e na parição, indicando que ovelhas de até seis dentes estão em fase de crescimento. As ovelhas Suffolk foram mais pesadas que as dos demais grupos raciais, com exceção ao grupo Hampshire Down quanto ao peso ao parto. As ovelhas Corriedale e Ile de France tiveram médias de peso semelhantes e foram mais leves que as Hampshire Down e Suffolk em todas as pesagens.

Nos dois anos de avaliação, houve diferença no peso das ovelhas ao início da suplementação e ao início e final da estação de acasalamento, não se observando diferenças

Tabela 2 - Médias ( \pm erro-padrão) de peso $(\mathrm{kg})$ das ovelhas durante o período de suplementação e ao parto de acordo com o tratamento, o tipo de parição, a idade, o grupo racial e o ano de experimentação

Table 2 - Means ( \pm standard error) for body weight $(\mathrm{kg})$ of ewes during the supplementation period and at lambing according to the treatment, parturition type, age, breed and year of experiment

\begin{tabular}{|c|c|c|c|c|}
\hline $\begin{array}{l}\text { Efeito } \\
\text { Effect }\end{array}$ & $\begin{array}{l}\text { Início suplementação } \\
\text { Beginning of supplementation }\end{array}$ & $\begin{array}{l}\text { Início estação de monta } \\
\text { Beginning of breeding season }\end{array}$ & $\begin{array}{c}\text { Final estação de monta } \\
\text { End of breeding season }\end{array}$ & $\begin{array}{l}\text { Parto } \\
\text { Lambing }\end{array}$ \\
\hline \multicolumn{5}{|c|}{ Tratamento (Treatment) } \\
\hline $\mathrm{T} 1$ & $49,43 \pm 1,66$ & $48,28 \pm 1,61$ & $51,89 \pm 1,63^{b}$ & $51,70 \pm 1,74$ \\
\hline $\mathrm{T} 2$ & $50,62 \pm 1,61$ & $51,03 \pm 1,56$ & $57,66 \pm 1,58^{\mathrm{a}}$ & $55,25 \pm 1,58$ \\
\hline T 3 & $48,48 \pm 1,62$ & $49,37 \pm 1,57$ & $55,70 \pm 1,59^{a}$ & $52,71 \pm 1,71$ \\
\hline $\operatorname{Pr}>F$ & 0,5119 & 0,3229 & 0,0091 & 0,1840 \\
\hline
\end{tabular}

Tipo de parição

Parturition type

Não parida (Not lambed)

Parto simples (Single lambing)

Parto gemelar (Twin lambing)

$\operatorname{Pr}>\mathrm{F}$

Idade (Age)

4 dentes (Teeth)

6 dentes (Teeth)

8 dentes (Teeth)

$\operatorname{Pr}>\mathrm{F}$

Grupo racial (Breed)

Corriedale

Hampshire

Ile de France

Suffolk

$\operatorname{Pr}>\mathrm{F}$
$49,41 \pm 2,20$
$48,32 \pm 1,25$
$50,88 \pm 1,77$
0,3644
$45,86 \pm 2,08^{\mathrm{b}}$
$48,82 \pm 1,90^{b}$
$53,85 \pm 1,23^{\mathrm{a}}$
0,0008

\begin{abstract}
$41,87 \pm 3,17^{\mathrm{c}}$
$51,45 \pm 1,21^{\mathrm{b}}$

$47,41 \pm 1,96^{\mathrm{c}}$

$57,31 \pm 2,02^{\mathrm{a}}$

0,0004
\end{abstract}

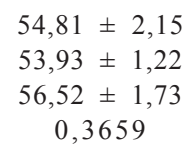

$52,04 \pm 2,04^{\mathrm{b}}$
$53,68 \pm 1,86^{\mathrm{b}}$
$59,53 \pm 1,21^{\mathrm{a}}$ 0,0006

\begin{abstract}
$49,47 \pm 2,13$
$48,15 \pm 1,21$

$1,06 \pm 1,7$ 0,2707
\end{abstract}

$$
\begin{aligned}
53,15 & \pm 1,26 \\
53,29 & \pm 1,76 \\
0,9403 &
\end{aligned}
$$

\begin{tabular}{|c|c|c|c|c|}
\hline 2001 & $47,58 \pm 1,46^{\mathrm{b}}$ & $47,51 \pm 1,41^{\mathrm{b}}$ & $52,00 \pm 1,43^{\mathrm{b}}$ & $52,42 \pm 1,40$ \\
\hline 2002 & $51,44 \pm 1,45^{\mathrm{a}}$ & $51,61 \pm 1,40^{\mathrm{a}}$ & $58,17 \pm 1,42^{\mathrm{a}}$ & $54,02 \pm 1,55$ \\
\hline
\end{tabular}

Ano (Year)

a, b, c: Médias seguidas de letras diferentes, na coluna, para o mesmo efeito, diferem a $5 \%$ de probabilidade.

T1: sem suplementação; T2: suplementação com milho triturado; T3: suplementação com mistura concentrada (75\% de milho triturado e $25 \%$ de farelo de soja). Means followed by different letters, within a column, for the same effect, differ at $5 \%$ of probability.

T1: Control, without supplementation; T2: Ewes supplemented with ground corn; T3: Ewes supplemented with a mixture of $75 \%$ ground corn and $25 \%$ soybean meal. 
nas médias de peso ao parto, o que sugere a ocorrência de efeito marcante do ambiente sobre os pesos dos animais.

Na Tabela 3 são apresentados os valores referentes aos ganhos de peso das ovelhas durante a suplementação e gestação. As ovelhas suplementadas (T2 e T3) ganharam mais peso $(\mathrm{P}<0,05)$ durante o período de suplementação, embora não tenha havido diferença significativa entre os pesos para os diferentes tratamentos ao início da estação de acasalamento (Tabela 2). Esses resultados foram semelhantes aos observados por Ribeiro et al. (2002).

Ovelhas que pariram gêmeos perderam mais peso entre o final da estação de monta e o parto, o que comprova maior requerimento nutricional para manutenção de uma gesta- ção gemelar, conforme indicação de tabelas de exigências nutricionais (NRC, 1985).

Ovelhas de seis dentes perderam peso do início da suplementação ao início da estação de acasalamento, com diferença significativa em relação às de oito dentes, porém, não houve diferenças entre as faixas etárias em outros períodos.

Os animais Ile de France perderam peso no período entre o início da suplementação e o início da estação de monta, o que ocasionou diferença significativa em relação aos dos grupos Hampshire Down e Suffolk. Os animais do grupo Corriedale também perderam peso, mas não o suficiente para determinar diferenças significativas com outros

Tabela 3 - Ganhos de peso médios $(\mathrm{kg})$ das ovelhas durante os períodos de suplementação (ganhos diários) e de gestação (ganhos totais durante todo o período), de acordo com o tratamento, o tipo de parição, a idade, o grupo racial e o ano de experimentação

Table 3 - Weight gains ( $\mathrm{kg}, \pm$ standard error) of ewes during supplementation (average daily gains) and gestation (total gain) according to the treatment, parturition type, age, breed and year of experiment

\begin{tabular}{|c|c|c|c|c|}
\hline Efeito & GMDISIM & GMDIMFM & GMDISFM & GMFMPA \\
\hline Effect & $A D G B S B B$ & $A D G B B F B$ & $A D G B S F B$ & \\
\hline
\end{tabular}

Tratamento

Treatment

\begin{tabular}{lcccc}
\hline T 1 & $-0,077 \pm 0,019^{\mathrm{b}}$ & $0,121 \pm 0,013^{\mathrm{b}}$ & $0,042 \pm 0,008^{\mathrm{b}}$ & $-0,241 \pm 1,100$ \\
T 2 & $0,028 \pm 0,018^{\mathrm{a}}$ & $0,221 \pm 0,013^{\mathrm{a}}$ & $0,117 \pm 0,008^{\mathrm{a}}$ & $-2,842 \pm 0,997$ \\
T 3 & $0,059 \pm 0,019^{\mathrm{a}}$ & $0,211 \pm 0,013^{\mathrm{a}}$ & $0,120 \pm 0,008^{\mathrm{a}}$ \\
Pr $>$ F & 0,0001 & 0,0001 & $-2,278 \pm 1,081$ & 0,0001 \\
\hline
\end{tabular}

Tipo de parição

Parturition type

Não parida (Not lambed)

Parto simples (Single lambing)

Parto gemelar (Twin lambing)

$\operatorname{Pr}>\mathrm{F}$

Idade (Age)

4 dentes (Teeth)

6 dentes (Teeth)

8 dentes (Teeth)

$\operatorname{Pr}>\mathrm{F}$

Grupo racial (Breed)

Corriedale

Hampshire

Ile de France

Suffolk

$\operatorname{Pr}>\mathrm{F}$

Ano (Year)

2001

2002

$\operatorname{Pr}>\mathrm{F}$
$0,004 \pm 0,025$
$-0,006 \pm 0,014$
$0,012 \pm 0,020$

0,6947
$0,178 \pm 0,018$
$0,192 \pm 0,010$
$0,182 \pm 0,014$

0,6452
$0,090 \pm 0,011$
$0,095 \pm 0,006$
$0,094 \pm 0,009$

0,9125
$-0,421 \pm 0,796^{\mathrm{a}}$

$-3,153 \pm 1,112^{b}$

0,0209
$0,103 \pm 0,010$
$0,081 \pm 0,009$
$0,095 \pm 0,006$
0,1757

$-1,113 \pm 1,281$

$-2,495 \pm 1,302$

$-1,754 \pm 0,786$

0,6937 \begin{tabular}{c}
0,2107 \\
\hline
\end{tabular}
$0,101 \pm 0,015$
$0,098 \pm 0,006$
$0,086 \pm 0,010$
$0,087 \pm 0,010$
0,5139

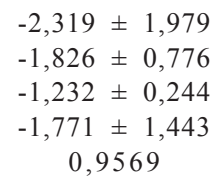

$-2,319 \pm 1,979$

0,9569

a, b: Médias seguidas de letras diferentes, na coluna, para o mesmo efeito, diferem a $5 \%$ de probabilidade.

GMDISIM: Ganho médio diário do início da suplementação até o início da estação de monta. GMDIMFM: Ganho médio diário do início ao final da estação de monta. GMDISFM: Ganho médio diário do início da suplementação até o final da estação de monta. GMFMPA: Ganho médio do final da estação de monta ao parto.

T1: sem suplementação. T2: suplementação com milho triturado. T3: suplementação com mistura concentrada ( $75 \%$ de milho triturado e $25 \%$ de farelo de soja).

Means followed by different letters, within a column, for the same effect, differ at $5 \%$ of probability.

ADGBSBB: Average daily gain from the beginning of supplementation to beginning of breeding season; ADGBBFB: Average daily gain from the beginning of breeding season to the end of breeding season; ADGBSFB: Average daily gain from the beginning of supplementation to the end of breeding season TGFBL: Total gain from the end of breeding season to lambing.

T1: Control, without supplementation; T2: Ewes supplemented with ground corn; T3: Ewes supplemented with a mixture of $75 \%$ ground corn and $25 \%$ soybean meal. 
grupos genéticos. Embora algumas diferenças nos ganhos de peso sejam estatisticamente significativas $(\mathrm{P}<0,05)$, não há uma explicação clara para esses resultados, ressaltando-se que as mudanças nos pesos foram de pequena magnitude.

Considerando o período de suplementação, as ovelhas ganharam mais peso em 2002 que em 2001. Entretanto, ao parto, em 2002 perderam peso (-3.776 g) em relação ao peso no final da estação de monta, ao passo que, em 2001, apresentaram ganho de $202 \mathrm{~g}$, determinando diferença altamente significativa $(\mathrm{P}<0,01)$ em relação ao ganho de peso. Todavia, as médias de peso ao parto foram similares entre os dois anos experimentais (Tabela 2), provavelmente porque, embora não tenha sido mensurada, visualmente observou-se maior disponibilidade forrageira no inverno de 2001, correspondente ao período de gestação.

O escore corporal das ovelhas dos tratamentos com suplementação foi superior $(\mathrm{P}<0,05)$ somente no final da estação de acasalamento (Tabela 4), assim como o peso vivo, sugerindo que o peso vivo não foi determinado pelo peso do trato gastroentérico, mas que houve deposição de tecidos (gordura e músculo) nas ovelhas sob suplementação. O escore das ovelhas no ano de 2001 foi maior que no ano 2002 $(\mathrm{P}<0,05)$ ao início da suplementação, mas essa diferença não se manteve até o final da estação de monta.

Os escores da condição corporal apresentaram relação direta com os pesos nas três pesagens, de modo que o maior peso determinou maior escore, reforçando a hipótese de maior deposição de tecidos nos animais. Apesar do maior peso corporal, no ano de 2002 (Tabela 2) as ovelhas apresentaram, em relação ao ano de 2001, menor escore da condição corporal no início da suplementação, o que significa que, provavelmente, elas eram maiores e tinham possibilidade de ganho de peso maior durante o período de suplementação, como observado (Tabela 3 ).

Tabela 4 - Escores corporais das ovelhas durante o período de suplementação, de acordo com os tratamentos, o tipo de parição, a idade, o grupo racial e o ano

Table 4 - Means ( \pm standard error) for body condition scores of ewes during the supplementation period according to the treatments, parturition type, age breed and year of experiment

\begin{tabular}{lccc}
\hline $\begin{array}{l}\text { Efeito } \\
\text { Effect }\end{array}$ & $\begin{array}{c}\text { Início suplementação } \\
\text { Beginning of supplementation }\end{array}$ & $\begin{array}{c}\text { Início estação de monta } \\
\text { Beginning of breeding season }\end{array}$ & $\begin{array}{c}\text { Final estação de monta } \\
\text { End of breeding season }\end{array}$ \\
\hline $\begin{array}{l}\text { Tratamento } \\
\text { Treatment }\end{array}$ & & & \\
T 1 & $2,41 \pm 0,13$ & $2,18 \pm 0,12$ & $2,37 \pm 0,13^{\mathrm{b}}$ \\
T 2 & $2,43 \pm 0,12$ & $2,43 \pm 0,12$ & $2,79 \pm 0,13^{\mathrm{a}}$ \\
T 3 & $2,35 \pm 0,12$ & $2,34 \pm 0,11$ & $2,81 \pm 0,13^{\mathrm{a}}$ \\
Pr $>\mathrm{F}$ & 0,8209 & 0,2316 & 0,0054 \\
\hline
\end{tabular}

Tipo de parição

Types of parturition

Não parida (Not lambed)

Parto simples (Single lambing)

Parto gemelar (Twin lambing)

$\operatorname{Pr}>\mathrm{F}$

Idade (Age)

4 dentes (Teeth)

6 dentes (Teeth)

8 dentes (Teeth)

$\operatorname{Pr}>\mathrm{F}$

Prepo racial (Breed)

Grupo racial (Breed)

$\begin{array}{lc}\text { Corriedale } & 2,24 \pm 0,24 \\ \text { Hampshire } & 2,50 \pm 0,09 \\ \text { Ile de France } & 2,55 \pm 0,15 \\ \text { Suffolk } & 2,30 \pm 0,15 \\ \text { Pr }>\text { F } & 0,4192\end{array}$

Ano (Year)

$2,42 \pm 0,13$

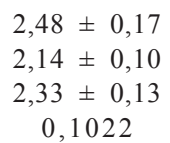

$2,66 \pm 0,17$

$2,63 \pm 0,10$

$2,68 \pm 0,14$

0,3956

0,1022

a,b: Médias seguidas de letras diferentes, na coluna, para o mesmo efeito, diferem a $5 \%$ de probabilidade.

T1: sem suplementação. T2: suplementação com milho triturado. T3: suplementação com mistura concentrada ( $75 \%$ milho triturado e $25 \%$ farelo de soja). Means followed by different letters, within a column, for the same effect, differ at $5 \%$ of probability.

T1: Control, without supplementation; T2: Ewes supplemented with ground corn; T3: Ewes supplemented with a mixture of $75 \%$ ground corn and $25 \%$ soybean meal. 
$\mathrm{Na}$ Tabela 5 constam os valores de desempenho reprodutivo das ovelhas. O flushing alimentar não determinou maior taxa de parição nem maior número de partos gemelares ( $\mathrm{P}>0,05)$, como observado por Ribeiro et al. (2002). Cumming (1977), trabalhando com raças selecionadas para a produção lã, verificou que o plano de nutrição antes e durante a estação de monta não afetou significativamente as taxas de ovulação. Esses resultados divergem, no entanto, dos observados por Gunn et al. (1979), que registraram maiores taxas de ovulação em ovelhas com escores corporais de 2,5, valor próximo da média (2,39) observada no início da suplementação. Segundo Robinson et al. (2002), o escore da condição corporal para se obter o desempenho reprodutivo máximo dos animais deve ser de 3,0 a 3,5, em uma escala de 5,0 pontos.

Embora as ovelhas que receberam suplementação alimentar (T2 e T3) tenham apresentado ganhos de peso e escores corporais maiores que as do tratamento sem suplementação (T1), apenas aquelas que consumiram milho triturado como suplemento alimentar exclusivo (T2) apre- sentaram melhor índice de natalidade $(\mathrm{P}<0,05)$. A razão deste resultado não é clara; provavelmente envolvia outros fatores não identificados ligados ao metabolismo nutricional. Molle et al. (1995) afirmam que os níveis de glicose, aminoácidos e insulina circulantes podem afetar a taxa de ovulação. Esses autores verificaram, em ovelhas que receberam farelo de soja, taxa de ovulação maior que naquelas que consumiram milho triturado, porém, não notaram diferenças no número de cordeiros nascidos por ovelha parida. Barioglio \& Rubiales de Barioglio(1994) verificaram que a suplementação energética em ovelhas Corriedale determinou taxa de natalidade de $180 \%$ (18 cordeiros nascidos em dez ovelhas expostas).

As ovelhas de oito dentes apresentaram maior $(\mathrm{P}<0,05)$ porcentagem de partos gemelares, implicando, conseqüentemente, maior índice de natalidade. Analla et al. (1998), estudando várias linhagens de Merino Espanhol, verificaram que ovelhas entre 6 e 8 anos de idade tiveram maior taxa de prolificidade que as mais jovens.

A taxa de parição foi maior nas ovelhas Corriedale, menor nas Suffolk e intermediária nas Ile de France e Hampshire

Tabela 5 - Número e porcentagem (entre parênteses) de ovelhas em reprodução (OR), paridas (OP), com partos simples ou gemelares, número de cordeiros nascidos (CN) e índice de natalidade (IN)

Table 5 - N Number and percentage (between parentheses) of ewes exposed (EE), lambed (EL), with single or twin lambing, number of lambs born (LB) and birth rate $(B R)$

\begin{tabular}{|c|c|c|c|c|c|c|}
\hline \multirow{3}{*}{$\begin{array}{l}\text { Efeito } \\
\text { Effect }\end{array}$} & \multirow{3}{*}{$\begin{array}{l}\mathrm{OR} \\
E E\end{array}$} & \multirow{3}{*}{$\begin{array}{c}\text { OP (taxa de parição) } \\
\text { EL (lambing rate) }\end{array}$} & \multirow{2}{*}{\multicolumn{2}{|c|}{$\begin{array}{l}\text { Tipo de parição } \\
\text { Type of parturition }\end{array}$}} & \multirow{3}{*}{$\begin{array}{l}\mathrm{CN} \\
L B\end{array}$} & \multirow{3}{*}{$\begin{array}{l}\mathrm{IN} \\
B R\end{array}$} \\
\hline & & & & & & \\
\hline & & & $\begin{array}{l}\text { Simples } \\
\text { Single }\end{array}$ & $\begin{array}{l}\text { Gemelar } \\
\text { Twin }\end{array}$ & & \\
\hline
\end{tabular}

Tratamento

Treatment

\begin{tabular}{lllcccc} 
T 1 & 34 & $28(82,35)$ & $19(67,86)$ & $9(32,14)$ & 37 & $1,088^{\mathrm{b}}$ \\
T 2 & 38 & $33(86,84)$ & $20(60,61)$ & $13(39,39)$ & 46 & $1,211^{\mathrm{a}}$ \\
T 3 & 40 & $34(85,00)$ & $25(73,53)$ & $9(26,47)$ & 43 \\
\hline Idade (Age) & & & & & & \\
& 25 & $22(88,00)$ & $18(81,82)^{\mathrm{a}}$ & $4(18,18)^{\mathrm{b}}$ & 26 \\
4 dentes (Teeth) & 24 & $19(79,17)$ & $15(78,95)^{\mathrm{a}}$ & $4(21,05)^{\mathrm{b}}$ & 23 & $1,040^{\mathrm{b}}$ \\
6 dentes (Teeth) & 63 & $54(85,71)$ & $31(57,41)^{\mathrm{b}}$ & $23(42,59)^{\mathrm{a}}$ & 77 \\
8 dentes (Teeth) & & & & $1,222^{\mathrm{a}}$ \\
\hline
\end{tabular}

Grupo racial

Breed

\begin{tabular}{|c|c|c|c|c|c|c|}
\hline Corriedale & 8 & $8(100,00)^{\mathrm{a}}$ & $5(62,50)$ & $3(37,50)$ & 11 & $1,375^{\mathrm{a}}$ \\
\hline Hampshire & 65 & $56(86,15)^{\mathrm{b}}$ & $39(69,64)$ & $17(30,36)$ & 73 & $1,123^{\mathrm{c}}$ \\
\hline Ile de France & 22 & $19(86,36)^{\mathrm{b}}$ & $12(63,16)$ & $7(36,84)$ & 26 & $1,182^{b}$ \\
\hline Suffolk & 17 & $12(70,59)^{\mathrm{c}}$ & $8(66,67)$ & $4(33,33)$ & 16 & $0,941^{\mathrm{d}}$ \\
\hline \multicolumn{7}{|l|}{ Ano } \\
\hline \multicolumn{7}{|l|}{ Year } \\
\hline 2001 & 63 & $57(90,48)^{\mathrm{a}}$ & $37(64,91)$ & $20(35,09)$ & 77 & $1,222^{\mathrm{a}}$ \\
\hline 2002 & 49 & $38 \quad(77,55)^{b}$ & $27(71,05)$ & $11(28,95)$ & 49 & $1,000^{\mathrm{b}}$ \\
\hline
\end{tabular}

a, b, c, d Médias seguidas de letras diferentes, na coluna, para cada efeito, diferem a $5 \%$ de probabilidade.

$a, b, c, d$ Means followed by different letters, within a column, for the same effect, differ at $5 \%$ of probability.

IN: Índice de natalidade (CN/OR) (NI: Natality index [LB/EE]).

Taxa de parição: OP/OR (Lambing rate: EL/EE).

T1: sem suplementação. T2: suplementação com milho triturado. T3: suplementação com mistura concentrada ( $75 \%$ de milho triturado e $25 \%$ de farelo de soja).

T1: control, without supplementation; T2: ewes supplemented with ground corn; T3: ewes supplemented with a mixture of $75 \%$ ground corn and $25 \%$ soybean meal. 
Down. Ressalta-se, no entanto, que foram avaliadas apenas oito ovelhas Corriedale, acasaladas com cordeiro Texel, e que, segundo Thomas et al. (1990), a heterose individual (do embrião) pode afetar positivamente o número de cordeiros nascidos. Esses fatores influenciaram os índices de natalidade, de modo que o grupo Corriedale apresentou maior taxa de natalidade, seguido pelo Hampshire Down, Ile de France e Suffolk, todos significativamente diferentes entre si. Demirören et al. (1995) estudaram raças norte-americanas e canadenses e constataram diferenças quanto à prolificidade, taxa de parição e incidência de partos gemelares entre as raças analisadas, comprovando a existência de diferenças entre grupos raciais quanto à fertilidade.

No ano de 2001, as ovelhas apresentaram taxas de parição e de natalidade maiores que em 2002. Considerando os resultados de ganho de peso e os escores de condição corporal, não é possível explicar estes resultados, que provavelmente decorreram de outros fatores não avaliados neste estudo. Demirören et al. (1995) observaram influência significativa do ano sobre os parâmetros reprodutivos das ovelhas e atribuíram este fato às influências ambientais.

\section{Conclusões}

A suplementação alimentar com milho triturado antes e durante a estação de monta pode influenciar positivamente o desempenho reprodutivo de ovelhas, elevando o número de cordeiros nascidos por ovelhas acasaladas. A idade e o grupo racial podem afetar significativamente o desempenho reprodutivo de rebanhos ovinos.

\section{Literatura Citada}

ABECIA, J.A.; FORCADA, F.; LOZANO, J.M. A preliminary report on the effect of dietary energy on prostaglandin $\mathrm{F}_{2}$ a production in vitro, interferon-tau synthesis by the conceptus, endometrial progesterone concentration on day 9 and 15 of pregnancy and associated rates of embryo wastage in ewes. Theriogenology, v.52, p.1203-1213, 1999

ANALlA, M.; MONTILlA, J.M.; SERRADILlA, J.M. Analyses of lamb weight and ewe litter size in various lines of Spanish Merino Sheep. Small Ruminant Research, v.29, p.255-259, 1998.

BARIOGLIO, C.; RUBIALES DE BARIOGLIO, S. Sincronizacion de celos y suplementacion energetica en ovejas. Archivos de Zootecnia, v.43, n.164, p.327-334, 1994.

BRANCA, A.; MOLLE, G.; SITZIA, M. et al. Short-term dietary effects on reproductive wastage after induced ovulation and artificial insemination in primiparous lactating Sarda ewes. Animal Reproduction Science, v.58, p.59-71, 2000.

CALDEIRA, R.M.; VAZ PORTUGAL, A. Condição corporal: conceitos, métodos de avaliação e interesse da sua utilização como indicador na exploração de ovinos. Revista Portuguesa de Ciências Veterinárias, v.93, n.526, p.95-102, 1998.

CUMMING, I. Relationship in the sheep of ovulation rate with live weight, breed, season and plane of nutrition. Australian
Journal of Experimental Agriculture and Animal Husbandry, v. 17, p.234-241, 1977.

DEMIRÖREN, E.; SHRESTHA, J.N.B.; BOYLAN, W.J. Breed and environmental effects on components of ewe productivity in terms of multiple births, artificial rearing and 8-month breeding cycles. Small Ruminant Research, v.16, p.239-249, 1995.

GUNN, R.G.; DONEY, J.M.; SMITH, W.F. Fertility in Cheviot ewes. 3 . The effect of level of nutrition before and after mating on ovulation rate and early embryo mortality in South Country Cheviot ewes in moderate condition at mating. Animal Production, v.29, p.25-31, 1979.

JAINUDEEN, M.R.; HAFEZ, E.S.E. Reproductive cycles: Sheep and goats. In: HAFEZ, E.S.E. (Ed.). 6.ed. Reproduction in farm animals. Philadelphia: Lea \& Febiger, 1993. p.330-342.

MAACK, R. Geografia física do estado do Paraná. 2.ed. Rio de Janeiro: José Olympio, 1981. 450p.

MOLLE, G.; BRANCA, A.; LIGIOS, S. et al. Effect of grazing background and flushing supplementation on reproductive performance in Sarda ewes. Small Ruminant Research, v.17, p.245-254, 1995 .

MOLLE, G.; LANDAU, S.; BRANCA, A. et al. Flushing with soybean meal can improve reproductive performances in lactating Sarda ewes on a mature pasture. Small Ruminant Research, v.24, n. 3, p.157-165, 1997.

MUKASA-MUGERWA, E.; LAHLOU-KASSI, A. Reproductive performance and productivity of Menz sheep in the Ethiopian highlands. Small Ruminant Research, v.17, p.167-177, 1995.

NOTTLE, M.B.; KLEEMANN, D.; GROSSER, T.I. et al. Evaluation of a nutritional strategy to increase ovulation rate in Merino ewes mated in late spring-early summer. Animal Reproduction Science, v.47, n.4, p.255-261, 1997.

NATIONAL RESEARCH COUNCIL - NRC. Nutrient requirements of sheep. 6.ed. Washington, D.C.: National Academy Press, 1985. 99p.

RIBEIRO, E.L.A.; SILVA, L.D.F.; MIZUBUTI, I.Y. et al. Desempenho produtivo de ovelhas acasaladas no verão e no outono recebendo ou não suplementação alimentar durante o acasalamento. Semina: Ciências Agrárias, v.23, n.1, p.35-44, 2002.

ROBINSON, J.J.; ROOKE, J.A.; McEVOY, T.G. Nutrition for conception and pregnancy. In: FREER, M.; DOVE, H. (Eds.). Sheep nutrition. Wallingford: CAB International, 2002. p. 189-211.

STATISTICAL ANALYSES SYSTEMS - SAS. SAS/STAT: user's guide. Cary: 1994. v.2, 846p.

SCARAMUZZI, R.J.; RADFORD, H.M. Factors regulating ovulation rate in the ewe. Journal of Reproduction and Fertility, v.69, p.353-367, 1983

SELAIVE-VILLARROEL, A.B. Manejo reprodutivo dos ovinos In: SIMPÓSIO PARANAENSE DE OVINOCULTURA, 3., 1986 , Guarapuava. Anais... Guarapuava: Associação Paranaense de Criadores de Ovinos, 1986. p.62-70.

SILVA, D.J.; QUEIROZ, A.C. Análise de alimentos: métodos químicos e biológicos. 3.ed. Viçosa, MG: Universidade Federal de Viçosa, 2002. 235p.

THOMAS, D.L.; NOTTER, D.R.; SHELTON, M. et al. Breeding and selection. In: SHEEP INDUSTRY DEVELOPMENT PROGRAM - SIDP (Ed.). Sheep production handbook. Denver: SIDP, 1990. p.1-64. 